\title{
Comparing Thoracic Extensive Laminoplasty (TELP) and Laminectomy in Treating Severe Thoracic Ligamentum Flavum Ossification: A Proposed Novel Technique and Case-Control Study
}

\author{
Jun Ma, ${ }^{1}$ Zhengyu Lu, ${ }^{1}$ Xin Zhou, ${ }^{1}$ Jia Yin, ${ }^{1}$ Enjie Xu, ${ }^{1}$ Heng Jiang, ${ }^{1}$ Xiao Ma, ${ }^{1}$ Yichen Meng, \\ Zhilin Li, ${ }^{2}$ Rui Gao, ${ }^{1}$ Tao Lin ${ }^{1 D}{ }^{1}$ and Xuhui Zhou ${ }^{1}{ }^{1}$ \\ ${ }^{1}$ Department of Orthopedics, Changzheng Hospital, Second Affiliated Hospital of Second Military Medical University, \\ 415 Fengyang Road, Shanghai, China 200003 \\ ${ }^{2}$ Department of Advertising, School of Journalism, Fudan University, 220 Handan Road, Shanghai, China 200000
}

Correspondence should be addressed to Tao Lin; tlinspine@outlook.com and Xuhui Zhou; xhzhouspine@163.com

Received 29 February 2020; Revised 14 January 2021; Accepted 10 April 2021; Published 21 April 2021

Academic Editor: Konstantinos Anagnostakos

Copyright ( 2021 Jun Ma et al. This is an open access article distributed under the Creative Commons Attribution License, which permits unrestricted use, distribution, and reproduction in any medium, provided the original work is properly cited.

\begin{abstract}
Objective. (1) To propose a novel technique named thoracic extensive laminoplasty (TELP) in curing severe thoracic ligamentum flavum ossification (STOLF) and (2) to compare outcomes between TELP and laminectomy in curing STOLF. Methods. Cases with fused or tuberous STOLF (Sato classification) treated from Jan 2015 to Jan 2017 were reviewed and divided into the TELP group (G1) and laminectomy group (G2) according to their surgical management. Data on demographics, complications, pre- and postoperative symptoms, residual spinal canal area (RSCA-1), residual spinal cord area (RSCA-2), modified Japanese Orthopedic Association score (mJOA), and health-related quality of life (HRQOL, based on the SF-36) were collected. Results. Fifty-nine G1 and sixty-two G2 patients were enrolled. No significant differences in demographic data or preoperative data of RSCA-1, RSCA-2, mJOA, or HRQOL were observed between the two groups $(p>0.05)$. Patients in G1 and G2 showed similar postoperative improvements in RSCA-1 and RSCA-2 at the final follow-up ( $p>0.05)$. However, patients in G1 showed higher postoperative improvements in $\mathrm{mJOA}(\mathrm{OR}=2.706,95 \% \mathrm{CI}: 1.279 \sim 5.727, p=0.008)$ at the final follow-up. Patients in $\mathrm{G} 1$ also showed higher postoperative improvements in HRQOL than patients in G2 $(p<0.05)$ at the final follow-up, and patients with more severe STOLF presented with better improvements in HRQOL in G1 $(p<0.05)$. Dural laceration and cerebrospinal fluid leakage were observed in seven G2 patients, and no complications were found in G1 patients after surgery. Conclusion. TELP is a novel, effective, and safer surgical technique in treating STOLF and could be a substitute for traditional laminectomy.
\end{abstract}

\section{Introduction}

Thoracic ossification of the ligamentum flavum (TOLF) is the most common cause of thoracic spinal stenosis, and it eventually leads to thoracic myelopathy, which was first reported by Tamaguchi et al. in 1960 [1]. TOLF occurs mainly in East Asia, including Korea, Japan, and China. Previous studies indicated that the incidence of TOLF was $21.8 \%$ (1090/4999) [2], 12\% (180/1500) [3], and 3.8\% (66/1736) [4] in Korea, Japan, and China, respectively. In China, it was reported that $63.9 \%$ of patients with chest symptoms were diagnosed with TOLF [5]. Meanwhile, the onset and progression of TOLF are insidious and chronic, which causes the diagnosis to frequently be missed and delayed. Most patients have already had a loss of functional gait when they come to see a doctor, and their prognosis is always poor $[6,7]$. Thus, how to effectively treat TOLF (especially severe TOLF (STOLF)) is still a challenge for doctors.

Sato et al. classified TOLF into five types according to its severity, and the fused and tuberous types were the most severe disorders. For STOLF, surgical management is the only effective treatment option $[8,9]$. Laminectomy is the traditional and currently recommended method for treating STOLF. The incidence of dural ossification in patients with STOLF is $43.4 \%$ [10], and complications of dural laceration and cerebrospinal fluid leakage are quite 
common during laminectomy, affecting approximately 10$32 \%$ of cases in a previous study [11]. Laminectomy combined with patch grafting is suggested for the treatment of STOLF with iatrogenic dural laceration, and laminectomy combined with floating the ossified dura by thinning it with a drill is applied for treating STOLF while avoiding damage to the dura. Both methods were thought to be effective in previous studies with a small sample size [12, 13]; however, considerations of infection, neurological damage, and operative complexity cannot be ignored. Thus, searching for a more effective and safer surgical method for the treatment of STOLF is of great value.

In our study, we aimed (1) to propose a novel surgical technique named thoracic extensive laminoplasty (TELP) for curing STOLF and (2) to verify the superior efficacy of TELP compared with traditional laminectomy (LT).

\section{Materials and Methods}

Cases of TOLF from Jan 2015 to Jan 2017 were reviewed in our hospital, and a case-controlled study was conducted. All patients were classified into two groups based on the surgical procedures they received: (1) patients who received TELP were grouped into G1, and (2) patients who received LT were grouped into G2.

\subsection{Inclusion Criteria. Inclusion criteria are as follows:}

(1) Age from 40 to 60 years old

(2) Followed up for at least 18 months

(3) Complete preoperative and postoperative anteroposterior thoracic plain X-ray, CT, and MRI

(4) Complete preoperative and postoperative evaluation of the modified Japanese Orthopedic Association score (mJOA) and health-related quality of life (HRQOL, based on the SF-36)

(5) All patients had fused or tuberous-type (Sato classification) TOLF, which was considered severe TOLF (STOLF).

\subsection{Exclusion Criteria}

(1) Patients with articular diseases of the axial skeleton, traumatic fractures of the spine, thoracic intervertebral disc herniation, and other ossifications of the spinal ligamentum were excluded

(2) Patients with cervical, lumbar, and sacral diseases were excluded

(3) Patients with diseases that influenced bone healing, such as osteoporosis, multiple myeloma, bone cancer, and bone tuberculosis, were also excluded.

2.3. Evaluation of Demographic Characteristics. Data on age, sex, body mass index (BMI), symptoms, blood loss, operating room time, follow-up time, postoperative dural laceration, and cerebrospinal fluid leakage (CSFL) were collected.
2.4. Image Evaluation. Patients with more than 50\% levels of tuberous TOLF were considered to have the tuberous type (T), and patients with more than $50 \%$ levels of fused TOLF were considered to have the fused type (F). Levels of STOLF, dural ossification or dural adhesion (comma sign or track sign), preoperative and postoperative residual spinal canal area (RSCA-1), and residual spinal cord area (RSCA-2) were evaluated based on CT and MRI.

The measurement method was performed according to Liu et al. [1] and is shown in Figure 1. If patients had more than one level of STOLF, we calculated the mean RSCA-1 (or RSCA-2) of all of the levels as the final result. The calculation of improvement was as follows: postoperative RSCA - 1 (or RSCA - 2) - preoperative RSCA - 1 (or RSCA - 2).

2.5. Neurological Evaluation. mJOA was collected before and after surgery or neurological assessment. The Hirabayashi recovery rate was calculated as follows [14]: recovery rate $=($ postoperative JOA score - preoperative $\mathrm{JOA}$ score $) /(11$ - preoperative JOA score $) \times 100 \%$.

2.6. Health-Related Quality of Life (HRQOL) Evaluation. HRQOL was evaluated before and after surgery based on the SF-36. Sections of physical functioning (PF), bodily pain $(\mathrm{BP})$, general health $(\mathrm{GH})$, and reported health transition (RHT) were included. The survey was in Chinese, and the normalized score was calculated according to Li et al. [15]. Normalized score $=($ Actual score - The lowest score of the section)/(The highest score of the section - The lowest score of the section $) \times 100$. Based on the calculated normalized score, the recovery rate was calculated as follows: recovery rate $=($ postoperative section score - preoperative section score $) /(100-$ preoperative section score $) \times 100 \%$. The lowest score of the section means the lowest score that patients can acquire in this section. The highest score of the section means the highest score that the patient can acquire in this section.

2.7. Statistical Analysis. We adopted the SPSS statistical analysis software version 13.0 (SPSS, Inc., Chicago, IL) for evaluation. Quantitative data were analyzed by Student's $t$-test, and classic data were analyzed by the Wilcoxon signed-rank test. Chi-squared analysis was also performed. Differences were considered significant when the $p$ value $<0.05$.

2.8. Ethics Statements. This research project was approved by the ethics department of our hospital. We obtained consent from all participants. All procedures were performed under the principles of the Declaration of Helsinki and relevant policies in China.

\section{Results}

3.1. Surgical Procedure of TELP. Endotracheal anesthesia was performed, and the patient was placed in the prone position before surgery. Neurophysiological monitoring involving somatosensory evoked potentials (SSEPs) and motor-evoked potentials (MEPs) was conducted throughout the entire process. A C-arm X-ray machine was used to target the influenced levels of the thoracic vertebrae 


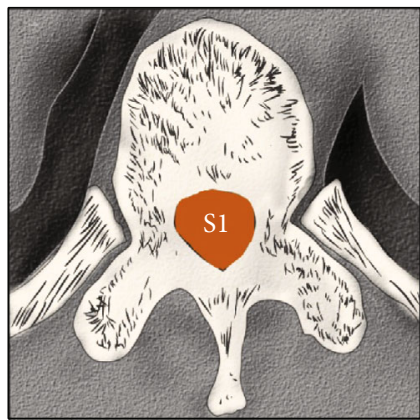

(a)

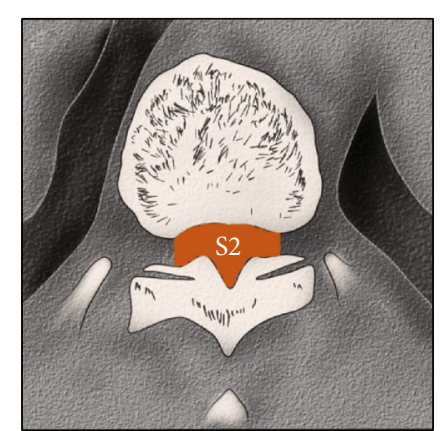

(b)

FIGURE 1: Illustration of residual spinal canal area (RSCA-1) and residual spinal cord area (RSCA-2). (a) The area of the normal spinal canal. (b) The area of the narrow spinal canal. RSCA-1 or RSCA-2: S2/S1; RSCA-1 was evaluated by CT; and RSCA-2 was evaluated by MRI.

pre- and intraoperatively. A posterior midline longitudinal incision was made, and procedures were adopted to completely expose the spinous processes, bilateral lamina, bilateral zygapophyseal joints, and the bilateral transverse process of the influenced levels. Tissues of the involved levels, including the spinous process, inferior portions of the superior lamina, superior portions of the inferior lamina, and interlamina ligamentum flavum, should be excised gently. Then, the normal boundaries of the thoracic spinal cord needed to be confirmed. Along the bilateral connections of the midpoint of the facet joints, which were taken as references, grinding was performed with a drill (Stryker, $4.0 \mathrm{~mm}$ ) at the lamina bilaterally. When the inner cortical bone was exposed, holes were drilled for the following fixation of the arch plate at the transverse process and lamina. Then, the grinding procedure was continued (Stryker, $3.0 \mathrm{~mm}$ ) until the lamina was isolated. Bipolar electrocoagulation and sterilized medical bone wax were used for hemostasis throughout the operation.

The connections of the soft tissues of the lamina were separated with a hooker nerve stripper from the caudal direction to the cephalad direction along the surface of the dural sac. Then, the arch plates were fixed bilaterally. Posterior fusion was carried out at the bilateral transverse process and the lamina with corticocancellous bone. To adjust the narrow part, an $\mathrm{O}$-arm $\mathrm{X}$-ray machine was used for space assessment of the spinal canal during the operation. All of the operative procedures are illustrated in Figures 2 and 3.

3.2. Surgical and Clinical Outcomes. A total of 59 patients in G1 (Figure 4) and 62 patients in G2 (Figure 5) were enrolled, and no patients were lost to follow-up. The average follow-up times were $19.4 \pm 2.1$ months and $18.9 \pm 2.8$ months in G1 and G2, respectively, and no significant differences were observed $(p=0.271)$. The average age, BMI, and sex proportions were similar between G1 and G2 $(p>0.05)$. In $\mathrm{G} 1$, the number of patients with lower limb (LB) numbness, LB spastic paraparesis, LB sensory deficits, gait instability, and urinary sphincter dysfunction was 42, $28,49,26$, and 18, respectively. In G2, 46, 33, 51, 29, and 22 patients had LB numbness, LB spastic paraparesis,
LB sensory deficits, gait instability, and urinary sphincter dysfunction, respectively. No significant constitutional differences were observed between G1 and G2 $(p>0.05)$. The average blood loss was $273.8 \pm 31.7 \mathrm{ml}$ in $\mathrm{G} 1$ and 281.4 $\pm 35.8 \mathrm{ml}$ in $\mathrm{G} 2$, and no significant differences were observed $(p=0.220)$. There were 7 patients with a dural laceration and 7 patients with cerebrospinal fluid leakage in G2; however, no patients with complications were found in G1 after the surgery (Table 1).

The levels of STOLF had no significant constitutional differences between the two groups $(p=0.325)$. The incidence of dural ossification or dural adhesion was $84.7 \%(50 / 59)$ in G1 and 83.9\% (52/62) in STOLF. Significant improvements in RSCA-1 were observed in both G1 $(p<0.0001)$ and G2 $(p<0.0001)$ after surgery, but no significant preoperative or postoperative differences in RSCA-1 were found between G1 and G2 $(p>0.05)$. Similar results were also found when we evaluated RSCA-2 (Table 2).

The average mJOA was $4.2 \pm 1.4$ in $\mathrm{G} 1$ and $4.3 \pm 1.7$ in $\mathrm{G} 2$, and no significant differences were found $(p=0.725)$ before surgery. The mean postoperative mJOA was $8.1 \pm$ 1.9 in G1 and $7.9 \pm 1.6$ in G2, and no significant differences were found $(p=0.532)$. The mJOA of patients in G1 and G2 all improved significantly $(p<0.05)$ after surgery. However, G1 had better neurological improvements $(\geq 50 \%$ recovery rate of $\mathrm{mJOA})$ than $\mathrm{G} 2(p=0.008, \mathrm{OR}=2.706,95 \% \mathrm{CI}$ : 1.279 5.727) (Table 3).

HRQOL was also assessed in G1 and G2 (Table 4). The preoperative PF, BP, GH, and RHT of HRQOL in G1 were $31.2 \pm 11.4,52.9 \pm 11.7,37.4 \pm 13.9$, and $28.9 \pm$ 8.7, respectively, and $31.7 \pm 12.1,53.1 \pm 10.8,37.2 \pm 10.7$, and $28.2 \pm 9.5$ in G2, respectively. No significant differences were observed between the two groups $(p>0.05)$. The postoperative PF, BP, GH, and RHT of HRQOL in G1 were $71.6 \pm 13.9,80.6 \pm 9.4,73.4 \pm 16.5$, and $70.7 \pm$ 11.1 , respectively, and $64.8 \pm 11.7,74.3 \pm 7.1,66.2 \pm 12.2$, and $66.4 \pm 10.9$ in G2, respectively, and the scores in G1 were higher than those in G2 $(p<0.05)$. The PF, BP, $\mathrm{GH}$, and RHT of the HRQOL of patients in G1 and G2 all improved significantly $(p<0.05)$ after surgery. In $\mathrm{G} 1$, tuberous-type patients had better PF improvements $(\geq 50 \%$ recovery rate of $\mathrm{PF}$ ) than fused-type patients $(p=0.007, \quad \mathrm{OR}=4.443,95 \%$ CI: $1.471 \sim 13.423)$. Similar 


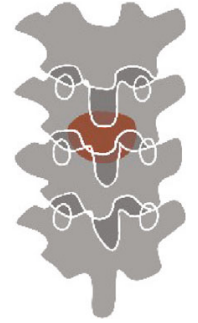

(a)

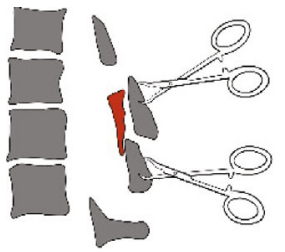

(e)

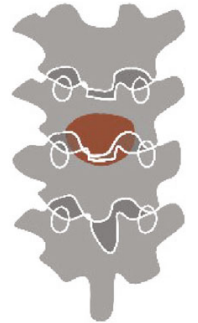

(b)

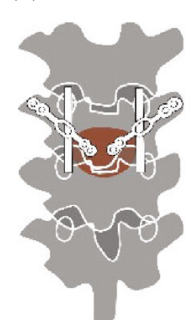

(f)

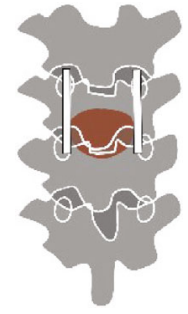

(c)

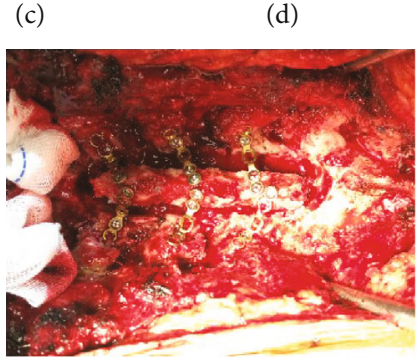

(g)

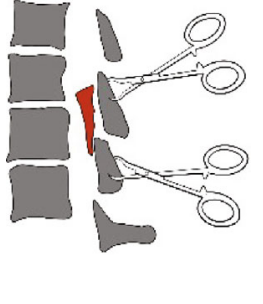

(d)$$
\text { g) }
$$

FIgURE 2: Illustration of the procedures of thoracic extensive laminoplasty (TELP) in a coronal view. (a) The thoracic spine with ossified ligamentum flavum (OLF in brown color). (b) Excision of the spinous process and portion of the lamina (not shown in the picture). (c) Bilateral grooves were made by grinding with a drill, and the lamina was isolated bilaterally. (d, e) Lifting the lamina (OLF in brown color) by towel forceps. (f) Fixation by arch plates and fusion with corticocancellous bone (not shown in the picture). (g) Intraoperative view of the TELP.

results were observed in $\mathrm{BP}, \mathrm{GH}$, and RHT of HRQOL. However, this interesting phenomenon was not found in G2.

\section{Discussion}

In our study, we proposed a novel technique of TELP for the treatment of STOLF. We also compared the radiographic, clinical, and HRQOL outcomes between patients who underwent TELP and those who underwent traditional laminectomy. Image evaluation showed that preoperative and postoperative RSCA-1 (or RSCA-2) had no significant differences between G1 and G2 $(p>0.05)$, and both techniques could provide effective and stable decompression of the spinal canal and cord. The average preoperative and postoperative mJOA values were not different between G1 and G2 $(p>0.05)$, and both techniques improved neurological function $(p<0.05)$. However, G1 had a better neurological recovery rate than $\mathrm{G} 2(p=0.08$, $\mathrm{OR}=2.706$ ). G1 had better HRQOL (based on the average PF, BP, GH, and RHT scores) than G2 $(p<0.05)$. In addition, G1 patients with more severe types of STOLF (tuberous type) tended to have better rehabilitation of HRQOL $(p<0.05)$; however, this phenomenon was not found in G2 patients.

Symptomatic thoracic myelopathy secondary to TOLF was the indication for surgical intervention, and sufficient decompression of the spinal canal was the key factor influencing the recovery efficacy. TOLF was classified into 5 types based on the severity of the ossified lesion configuration: lateral type, extended type, enlarged type, fused type, and tuberous type. Posterior decompressive surgery was recommended for TOLF, and the laminectomy technique was recommended. It was also suggested that en bloc laminectomy was suitable for the lateral and extended types, and separating laminectomy was ideal for thickening the tuberous type [13].

A previous radiology study also showed that laminectomy could provide significant improvements in canal diameter in curing TOLF $(15.1 \pm 6.8 \%, p<0.05)$ [16]. However, laminectomy has two main inevitable limitations, especially for STOLF. First, dural laceration combined with cerebrospinal fluid leakage, which could lead to cerebrospinal fluid pseudocysts, respiratory obstruction, wound dehiscence, and meningitis, cannot be ignored [17, 18], and dural ossification (DO) or dural adhesion (DA) is the reason for these complications. DO or DA is commonly seen in STOLF, and the reported incidence ranges from $22 \%$ to $62 \%$ [19-21]. Our data showed that the incidence was $84.7 \%(50 / 59)$ in G1 and $83.9 \%(52 / 62)$ in STOLF. Thus, some researchers have suggested that floating ossified lesions of the dural sac with a high-speed drill to abrade them until paper thin would benefit this situation [22]. However, this results in a lesser extent of spinal decompression, and there is still a high risk of iatrogenic dural tears. Second, laminectomy and excessive resection of the facet joints would cause structural instability, especially for multiple levels of STOLF [23]. It was reported that TOLF is located in multiple segments, tandem ossification is common, and its incidence is $33.3 \%$ to $55.1 \%$ [24, 25]. Structural instability would cause increased kyphotic deformity of the spine, which would lead to late neurological deterioration. Thus, laminoplasty was proposed. However, laminoplasty was not suitable for STOLF, as this 


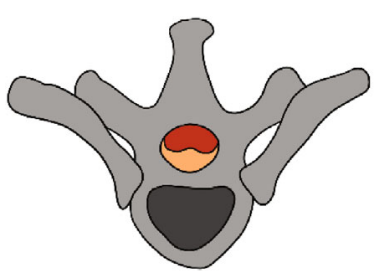

(a)

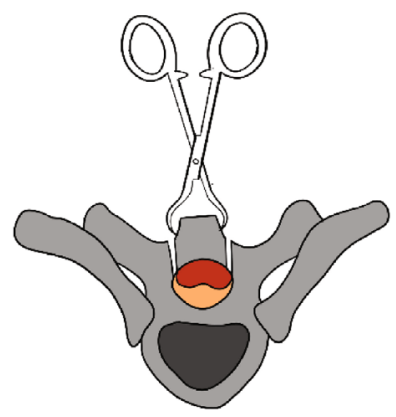

(c)

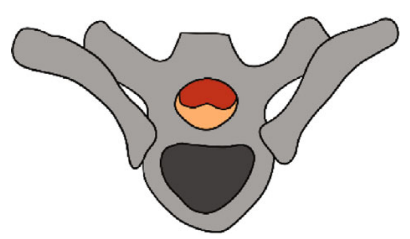

(b)

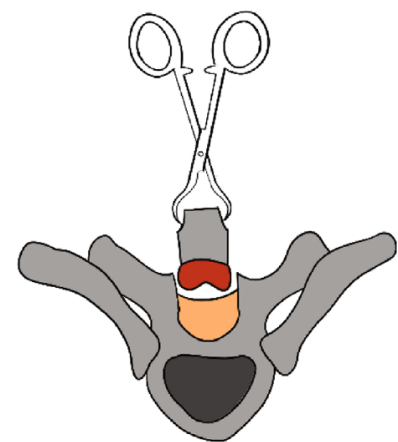

(d)

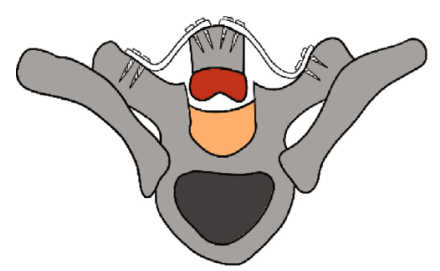

(e)

FIgURE 3: Illustration of the procedures of thoracic extensive laminoplasty (TELP) in a cross-section view. (a) The level of vertebra with TOLF. (b) Excision of the spinous process and portion of the lamina (not shown in the picture). (c) Bilateral grooves were made by grinding with a drill, and the lamina was isolated bilaterally. (d) Lifting the lamina by towel forceps. (e) Fixation by arch plates and fusion with corticocancellous bone (not shown in the picture).

technique could only achieve limited decompression without removal of the ossified ligamentum flavum. Laminectomy with fusion also remains controversial. Therefore, seeking a more effective surgical technique is urgent.

Our study proposed a novel surgical technique for STOLF called TELP. This technique had similar decompressive efficacy as laminectomy (with no significant differences in RSCA-1 and RSCA-2 between G1 and G2). No complications of dural laceration or cerebrospinal fluid leakage were observed, while 7 patients with dural laceration and cerebrospinal fluid leakage were found in the laminectomy group. Increased kyphotic deformity of the spine was not observed in G1 at the final follow-up.

To achieve these goals, the technique has several benefits. First, the isolated lamina could be elevated according to the decompressive demand, which could provide a sufficient volume of spinal canal. In addition, we did not resect the ossified lesions that adhered to the lamina and the dural sac during the procedure. Thus, the suitably and gently lifting force on the lamina would not cause dural tears, and sufficient decompression of the spinal cord would be achieved. Moreover, arch plate placement combined with bone grafting and the residual facet joint reconstructed the posterior column of the spine, which provided sufficient stability to avoid kyphotic deformity of the spine. In addition, the ossified lesion that we did not resect would not cause a second compression, and spontaneous reduction (or atrophy) was observed during the follow-up. Many studies have indicated the same phenomenon, and it is believed that pulsations of the thecal sac and venous plexus are responsible [26-28].

Previous studies demonstrated that the average recovery rate of neurological function based on mJOA was $40 \%$ to $50 \%$ after laminectomy in TOLF, which indicated fair rehabilitation [29-31]. Our studies also showed similar results for the reported outcomes in G2. No obvious differences in the average preoperative and postoperative mJOA were found between G1 and G2 $(p>0.05)$. Interestingly, we found that the outcome of neurological function in G1 was superior to that in G2 when we compared the number of patients with a $\geq 50 \% \mathrm{mJOA}$ recovery rate between G1 and G2. These results indicated that TELP might be more suitable for STOLF than laminectomy, which could be explained by the less invasive procedure of TELP that decreased the risk of neurologic damage. In addition, the spinal cord in the thoracic spine has worse vascularity and occupies a larger proportion of the spinal 


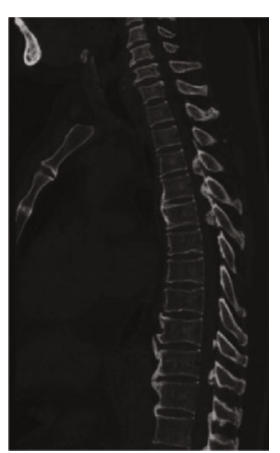

(a)

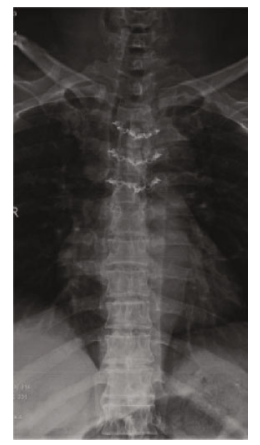

(c)

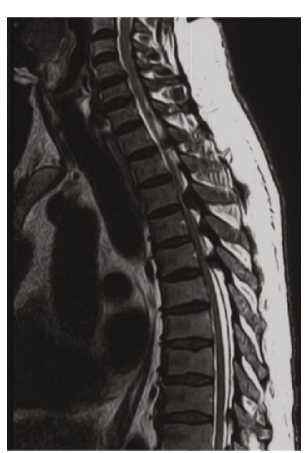

(b)

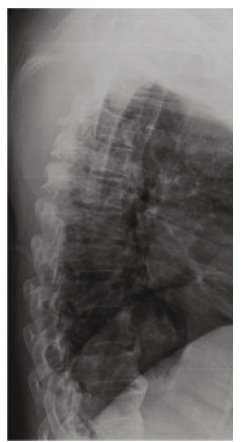

(d)

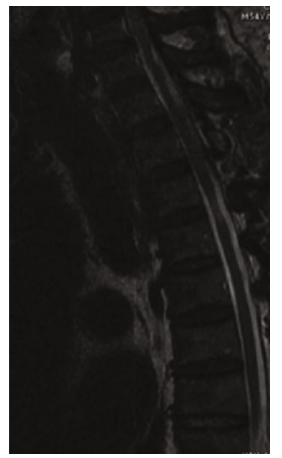

(e)

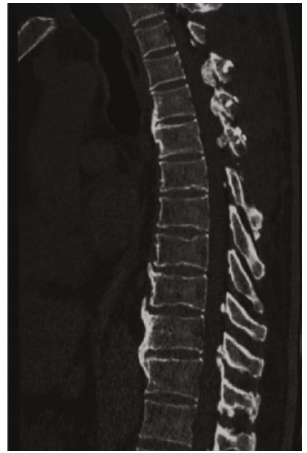

(f)

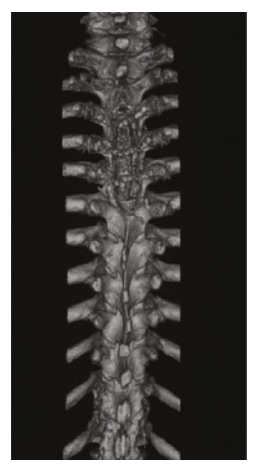

(g)

Figure 4: A 45-years old patient with STOLF from T3 to T5 levels who received TELP. (a) Preoperative CT. (b) Preoperative MRI. (c, d) Postoperative standing anteroposterior X-ray at final follow-up. (e) Postoperative MRI at final follow-up. (f) Postoperative CT at final follow-up. (g) Postoperative three-dimensional CT reconstruction at final follow-up.

canal, making it more susceptible to damage by a more invasive procedure [32]. Additional studies are needed for confirmation.
Our study also included an evaluation of the HRQOL of the patients based on the SF-36. To our knowledge, this was the first study to use a patient-reported scale as a part of 


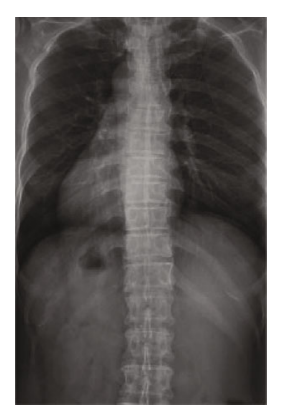

(a)

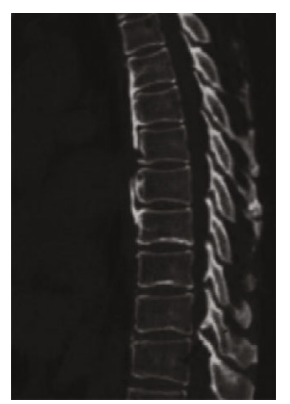

(c)

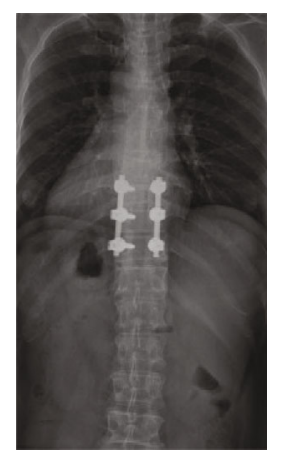

(e)

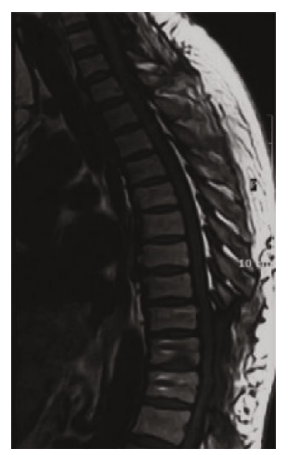

(g)

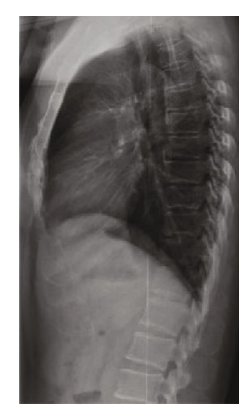

(b)

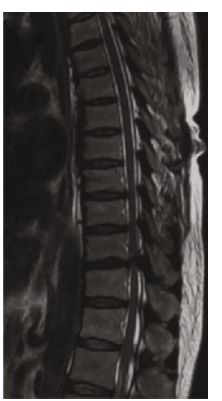

(d)

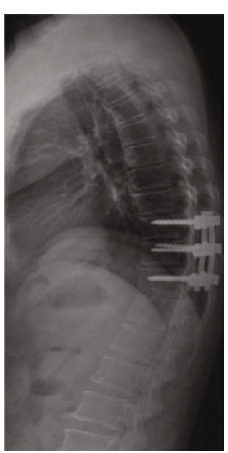

(f)

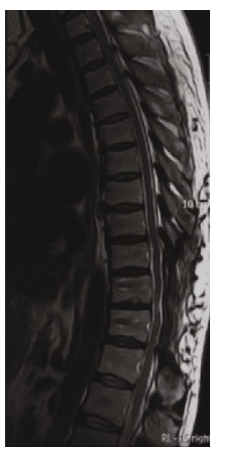

(h)

FIGURE 5: A 49-year-old patient with STOLF from T9 to T11 levels who received laminectomy. (a, b) Preoperative standing anteroposterior Xray. (c) Preoperative CT. (d) Preoperative MRI. (e, f) Postoperative standing anteroposterior X-ray at final follow-up. (g, h) Postoperative MRI at final follow-up.

the assessment of surgical efficacy. For PF, BP, GH, and RHT, both G1 and G2 had significant improvements after surgery, but G1 had better recovery than G2. These results indicated that patients who underwent TELP had better recovery of sensation than those who underwent laminectomy. This was an interesting phenomenon, and we supposed that this could be attributed to the less invasive procedure and no surgical complications in G1. Another interesting phenomenon was that patients with more severe TOLF conditions presented with better recovery of sensation in G1, while this phenomenon was not observed in G2. We supposed that preoperative expectations were the main reason for this 
TABLE 1: Demographic characteristics (mean \pm SD or $n$ ).

\begin{tabular}{|c|c|c|c|}
\hline Item & G1 & G2 & $p$ value \\
\hline \multirow{2}{*}{ Sex, $n$} & Male: 35 & Male: 39 & \multirow{2}{*}{0.686} \\
\hline & Female: 24 & Female: 23 & \\
\hline Age, years old & $52.2 \pm 4.7$ & $51.8 \pm 7.3$ & 0.722 \\
\hline BMI, $\mathrm{kg} / \mathrm{m}^{2}$ & $24.1 \pm 2.6$ & $23.7 \pm 3.2$ & 0.453 \\
\hline \multicolumn{4}{|l|}{ Symptom, $n(\%)$} \\
\hline LB numbness & $42(71.2)$ & $46(74.2)$ & 0.710 \\
\hline LB spastic paraparesis & $28(47.5)$ & $33(53.2)$ & 0.526 \\
\hline LB sensory deficits & $49(83.1)$ & $51(82.3)$ & 0.908 \\
\hline Gait instability & $26(44.1)$ & $29(46.8)$ & 0.765 \\
\hline Urinary sphincter dysfunction & $18(30.5)$ & $22(35.5)$ & 0.561 \\
\hline Blood loss, $\mathrm{ml}$ & $273.8 \pm 31.7$ & $281.4 \pm 35.8$ & 0.220 \\
\hline Operating room time, hours & $3.5 \pm 1.8$ & $3.8 \pm 1.6$ & 0.334 \\
\hline Follow-up time, months & $19.4 \pm 2.1$ & $18.9 \pm 2.8$ & 0.271 \\
\hline Dural laceration, $n$ & 0 & 17 & -- \\
\hline Cerebrospinal fluid leakage, $n$ & 0 & 17 & -- \\
\hline
\end{tabular}

BMI: body mass index; LB: lower limbs.

TABLE 2: Image evaluation (mean \pm SD or $n$ ).

\begin{tabular}{lccc}
\hline Item & $\mathrm{G} 1$ & $\mathrm{G} 2$ & $p$ value \\
\hline Single level, $n$ & 10 & 15 & 0.325 \\
$\geq 2$ levels, $n$ & 49 & 47 & \\
DO or DA, $n$ & 50 & 52 & 0.895 \\
RSCA-1 & & & \\
$\quad$ Preoperation, \% & $37.9 \pm 2.5$ & $38.1 \pm 3.2$ & 0.703 \\
$\quad$ Postoperation, \% & $78.2 \pm 5.3$ & $78.1 \pm 4.8$ & 0.913 \\
$\quad$ value & $<0.0001$ & $<0.0001$ & -- \\
$\quad$ Improvement, \% & $40.3 \pm 3.9$ & $40.0 \pm 4.1$ & 0.681 \\
RSCA-2 & & & \\
$\quad$ Preoperation, \% & $37.2 \pm 2.8$ & $37.6 \pm 2.6$ & 0.417 \\
$\quad$ Postoperation, \% & $79.4 \pm 4.4$ & $78.8 \pm 3.9$ & 0.428 \\
$\quad$ value & $<0.0001$ & $<0.0001$ & -- \\
$\quad$ Improvement, \% & $42.2 \pm 3.3$ & $41.2 \pm 2.9$ & 0.079 \\
\hline
\end{tabular}

Postoperation: postoperation at final follow-up. DO or DA: dural ossification or dural adhesion.

TABLE 3: Neurological evaluation based on mJOA (mean \pm SD or $n)$.

\begin{tabular}{lccc}
\hline Item & G1 & G2 & $p$ value \\
\hline Preoperation & $4.2 \pm 1.4$ & $4.3 \pm 1.7$ & 0.725 \\
Postoperation & $8.1 \pm 1.9$ & $7.9 \pm 1.6$ & 0.532 \\
$p$ value & $<0.0001$ & $<0.0001$ & -- \\
$\geq 50 \%{ }^{\Delta}$ & 31 & 18 & \\
$<50 \%{ }^{\Delta}$ & 28 & 44 & 0.008 \\
OR $(95 \% \mathrm{CI})$ & $2.706(1.279 \sim 5.727)$ & -- \\
\hline
\end{tabular}

${ }^{\Delta}$ Recovery rate. Postoperation: postoperation at final follow-up. phenomenon. Patients with tuberous-type TOLF suffer from more severe symptoms, and a small improvement might provide them with more recovery satisfaction [33]. In addition, TELP is less invasive, and no complications were observed, which might be another reason for this finding. Additional studies are necessary.

Recently, Sun et al. [28] reported a new surgical technique for TOLF that used transverse connectors and surgical lines to build a "bridge crane" to suspend the lamina for the purpose of decompression. The extent of decompression was controlled by the extent of tightening of the surgical lines. This was absolutely an innovation in the surgical community; however, three main limitations should be considered. First, the structural stability was of concern. If the surgical lines are not tightened after adhering to the connector, the isolated lamina attached to the lines will shake, which might cause damage to the dural sac. Actually, the surgical lines could not always be tightened after adhering to the connector because of the consideration of not tearing the dural sac. Second, the surgeon needed to drill a tunnel into the lamina for the surgical line, which was complicated and had a risk of puncturing the dural sac. Finally, the space of the thoracic vertebral canal and pedicle was narrow, and the use of pedicle screws would cause insufficient decompression. In addition, the cost of TELP is lower than that of bridge cranes. Our novel technique of TELP with an arch plate as the fixation material overcame all of the above limitations. Surgeons could control the decompression by changing the angle of the arch plate, which is safe and reliable.

However, several limitations of this study exist. First, the sample size was small, and the follow-up time was limited, which would decrease the reliability and validity of the study. Second, the pulsative movement of the dura mater could not be directly observed, which made intraoperative judgment of decompression of the spinal cord difficult. Third, it was 
TABLE 4: HRQOL of the patients based on SF-36 (mean \pm SD or $n)$.

\begin{tabular}{|c|c|c|c|c|c|c|c|c|}
\hline \multirow[t]{2}{*}{ Item } & \multicolumn{2}{|c|}{$\mathrm{PF}$} & \multicolumn{2}{|l|}{$\mathrm{BP}$} & \multicolumn{2}{|c|}{$\mathrm{GH}$} & \multicolumn{2}{|c|}{ RHT } \\
\hline & G1 & G2 & G1 & G2 & G1 & G2 & G1 & G2 \\
\hline Pre & $31.2 \pm 11.4$ & $31.7 \pm 12.1$ & $52.9 \pm 11.7$ & $53.1 \pm 10.8$ & $37.4 \pm 13.9$ & $37.2 \pm 10.7$ & $28.9 \pm 8.7$ & $28.2 \pm 9.5$ \\
\hline Post & $71.6 \pm 13.9$ & $64.8 \pm 11.7$ & $80.6 \pm 9.4$ & $74.3 \pm 7.1$ & $73.4 \pm 16.5$ & $66.2 \pm 12.2$ & $70.7 \pm 11.1$ & $66.4 \pm 10.9$ \\
\hline $\mathrm{D} 1(\mathrm{C} 1 \mathrm{C}, \mathrm{U})$ & \multicolumn{2}{|c|}{ Pre: 0.816} & \multicolumn{2}{|c|}{ Pre: 0.922} & \multicolumn{2}{|c|}{ Pre: 0.268} & \multicolumn{2}{|c|}{ Pre: 0.674} \\
\hline$P 1(G 1 / G 2)$ & \multicolumn{2}{|c|}{ Post: 0.004} & \multicolumn{2}{|c|}{ Post: $<0.0001$} & \multicolumn{2}{|c|}{ Post: 0.007} & \multicolumn{2}{|c|}{ Post: 0.034} \\
\hline \multirow{3}{*}{ P2 (pre/post) } & \multicolumn{2}{|c|}{ G1: $<0.0001$} & \multicolumn{2}{|c|}{$\mathrm{G} 1:<0.0001$} & \multicolumn{2}{|c|}{ G1: $<0.0001$} & \multicolumn{2}{|c|}{$\mathrm{G} 1:<0.0001$} \\
\hline & \multicolumn{2}{|c|}{$\mathrm{G} 2:<0.0001$} & \multicolumn{2}{|c|}{ G2: $<0.0001$} & \multicolumn{2}{|c|}{ G2: $<0.0001$} & \multicolumn{2}{|c|}{$\mathrm{G} 2:<0.0001$} \\
\hline & $\mathrm{T} / \mathrm{F}$ & $\mathrm{T} / \mathrm{F}$ & $\mathrm{T} / \mathrm{F}$ & $\mathrm{T} / \mathrm{F}$ & $\mathrm{T} / \mathrm{F}$ & $\mathrm{T} / \mathrm{F}$ & $\mathrm{T} / \mathrm{F}$ & $\mathrm{T} / \mathrm{F}$ \\
\hline$\geq 50 \%^{\Delta}$ & $23 / 11$ & $10 / 15$ & $28 / 11$ & $13 / 9$ & $26 / 12$ & $12 / 13$ & $22 / 12$ & $11 / 14$ \\
\hline$<50 \%^{\Delta}$ & $8 / 17$ & $20 / 17$ & $3 / 17$ & $17 / 23$ & $5 / 16$ & $18 / 19$ & $9 / 16$ & $19 / 18$ \\
\hline$p$ value & 0.007 & 0.277 & $<0.0001$ & 0.211 & 0.001 & 0.960 & 0.029 & 0.570 \\
\hline OR & 4.443 & - & 14.424 & - & 6.933 & - & 3.259 & - \\
\hline (95\% CI) & $(1.471 \sim 13.423)$ & - & $(3.516 \sim 59.181)$ & - & $(2.057 \sim 23.368)$ & - & $(1.109 \sim 9.576)$ & - \\
\hline
\end{tabular}

PF: physical functioning; BP: bodily pain; GH: general health; RHT: reported health transition; Pre: preoperation; Post: postoperation at final follow-up; T: tuberous type of STOLF; F: fused-type PF STOLF; ${ }^{4}$ recovery rate.

difficult to achieve hemostasis directly, as the structures inside the canal were not visible.

\section{Conclusion}

Our study proposed a novel and effective TELP technique for STOLF. We found that this technique was equivalent to laminectomy in canal and spinal cord decompression. However, our technique was better for neurological recovery, and patients also experienced better HRQOL recovery. TELP could also decrease the risk of dural tears and late-onset kyphotic deformities of the spine, especially for multisegmental STOLF. We hope that our TELP will benefit patients as well as surgeons. Our future research direction will be to conduct a large-scale cohort study for additional evaluation of this technique.

\section{Data Availability}

All the data associated with this article is deposited in the table part (Tables 1 to 4 ).

\section{Ethical Approval}

The research project was approved by the ethics department of Shanghai Changzheng Hospital, Shanghai. We have consensus with all participants. All the procedures were done under the Declaration of Helsinki and relevant policies in China.

\section{Conflicts of Interest}

All authors declared that they have no potential conflict of interest.

\section{Authors' Contributions}

Jun Ma, Zhengyu Lu, Xin Zhou, and Jia Yin contributed equally to this work.

\section{Acknowledgments}

The received foundations are as follows: Chenguang Program of Shanghai Municipal Education Commission (Grant No. 14CG37); Science Research Project of Science and Technology Commission of Shanghai Municipality (Grant No. 18411964800); Youth Research Project of Shanghai Municipal Health Bureau (Grant No. 2012Y146); and Military Medical Science and Technology Youth Training Project (Grant No. 18QNP015). This research has not received any funding. Thanks are due to Zhilin Li for drawing the pictures which were used for the illustration of surgical procedures. The manuscript submitted does not contain information about medical device(s)/drug(s).

\section{References}

[1] F.-b. Feng, C.-g. Sun, and Z.-q. Chen, "Progress on clinical characteristics and identification of location of thoracic ossification of the ligamentum flavum," Orthopaedic Surgery, vol. 7, no. 2, pp. 87-96, 2015.

[2] S.-I. Kim, K.-Y. Ha, J.-W. Lee, and Y.-H. Kim, "Prevalence and related clinical factors of thoracic ossification of the ligamentum flavum-a computed tomography-based cross-sectional study," The Spine Journal, vol. 18, no. 4, pp. 551-557, 2018.

[3] T. Fujimori, T. Watabe, Y. Iwamoto, S. Hamada, M. Iwasaki, and T. Oda, "Prevalence, concomitance, and distribution of ossification of the spinal ligaments results of whole spine CT scans in 1500 Japanese patients," Spine (Phila Pa 1976), vol. 41, no. 21, pp. 1668-1676, 2016.

[4] J. J. Guo, K. D. K. Luk, J. Karppinen, H. Yang, and K. M. C. Cheung, "Prevalence, distribution, and morphology of ossification of the ligamentum flavum: a population study of one thousand seven hundred thirty-six magnetic resonance imaging scans," Spine (Phila Pa 1976), vol. 35, no. 1, pp. 51-56, 2010.

[5] N. Lang, H. S. Yuan, H. L. Wang et al., "Epidemiological survey of ossification of the ligamentum flavum in thoracic spine: CT 
imaging observation of 993 cases," European Spine Journal, vol. 22, no. 4, pp. 857-862, 2013.

[6] D. K. Ahn, S. Lee, S. H. Moon, K. H. Boo, B. K. Chang, and J. I. Lee, "Ossification of the ligamentum flavum," Asian Spine Journal, vol. 8, no. 1, pp. 89-96, 2014.

[7] K.-C. Kang, C.-S. Lee, S.-K. Shin, S.-J. Park, C.-H. Chung, and S.-S. Chung, "Ossification of the ligamentum flavum of the thoracic spine in the Korean population," Journal of Neurosurgery. Spine, vol. 14, no. 4, pp. 513-519, 2011.

[8] S. Nayak, "P299 - Thoracic Myelopathy Due to Ossified Ligamentum Flavum, Operative Manangement and Outcome," Global Spine Journal, vol. 7, 2_suppl, pp. 190S-373S, 2017.

[9] P. Trivedi, S. Behari, L. Paul, D. Banerji, V. K. Jain, and D. K. Chhabra, "Thoracic myelopathy secondary to ossified ligamentum flavum," Acta Neurochirurgica, vol. 143, no. 8, pp. 775-782, 2001.

[10] B. Li, G. Qiu, S. Guo et al., "Dural ossification associated with ossification of ligamentum flavum in the thoracic spine: a retrospective analysis," BMJ Open, vol. 6, no. 12, pp. e013887e013887, 2016.

[11] Z. M. Zhong, Q. Wu, T. T. Meng et al., "Clinical outcomes after decompressive laminectomy for symptomatic ossification of ligamentum flavum at the thoracic spine," Journal of Clinical Neuroscience, vol. 28, pp. 77-81, 2016.

[12] J. Sun, C. Zhang, G. Ning et al., "Surgical strategies for ossified ligamentum flavum associated with dural ossification in thoracic spinal stenosis," Journal of Clinical Neuroscience, vol. 21, no. 12, pp. 2102-2106, 2014.

[13] F. Li, Q. Chen, and K. Xu, "Surgical treatment of 40 patients with thoracic ossification of the ligamentum flavum," Journal of Neurosurgery. Spine, vol. 4, no. 3, pp. 191-197, 2006.

[14] B. J. Lee, J. H. Park, S. R. Jeon, S. C. Rhim, and S. W. Roh, "Clinically significant radiographic parameter for thoracic myelopathy caused by ossification of the ligamentum flavum," European Spine Journal, vol. 28, no. 8, pp. 18461854, 2019.

[15] L. Li, H. M. Wang, and Y. Shen, "Chinese SF-36 Health Survey: translation, cultural adaptation, validation, and normalisation," Journal of Epidemiology \& Community Health, vol. 57, no. 4, pp. 259-263, 2003.

[16] A. V. Sanghvi, H. S. Chhabra, A. A. Mascarenhas, V. K. Mittal, and G. M. Sangondimath, "Thoracic myelopathy due to ossification of ligamentum flavum: a retrospective analysis of predictors of surgical outcome and factors affecting preoperative neurological status," European Spine Journal, vol. 20, no. 2, pp. 205-215, 2011.

[17] M. H. Khan, J. Rihn, G. Steele et al., "Postoperative management protocol for incidental dural tears during degenerative lumbar spine surgery: a review of 3, 183 consecutive degenerative lumbar cases," Spine (Phila Pa 1976), vol. 31, no. 22, pp. 2609-2613, 2006.

[18] M. Takahata, M. Ito, K. Abumi, Y. Kotani, H. Sudo, and A. Minami, "Clinical results and complications of circumferential spinal cord decompression through a single posterior approach for thoracic myelopathy caused by ossification of posterior longitudinal ligament," Spine (Phila Pa 1976), vol. 33, no. 11, pp. 1199-1208, 2008.

[19] Z. Li, D. Ren, Y. Zhao et al., "Clinical characteristics and surgical outcome of thoracic myelopathy caused by ossification of the ligamentum flavum: a retrospective analysis of 85 cases," Spinal Cord, vol. 54, no. 3, pp. 188-196, 2016.
[20] K. B. Hamouda, H. Jemel, S. Haouet, and M. Khaldi, "Thoracic myelopathy caused by ossification of the ligamentum flavum: a report of 18 cases," Journal of Neurosurgery, vol. 99, no. 2, pp. 157-161, 2003.

[21] N. Miyakoshi, Y. Shimada, T. Suzuki et al., "Factors related to long-term outcome after decompressive surgery for ossification of the ligamentum flavum of the thoracic spine," Journal of Neurosurgery, vol. 99, no. 3, pp. 251-256, 2003.

[22] B. He, L. Yan, Z. Xu, H. Guo, T. Liu, and D. Hao, “Treatment strategies for the surgical complications of thoracic spinal stenosis: a retrospective analysis of two hundred and eighty three cases," International Orthopaedics, vol. 38, no. 1, pp. 117-122, 2014.

[23] T. Wang, C. Yin, D. Wang, S. Li, and X. Chen, "Surgical technique for decompression of severe thoracic myelopathy due to tuberous ossification of ligamentum flavum," Clinical Spine Surgery: A Spine Publication, vol. 30, no. 1, pp. E7-E12, 2017.

[24] J. Kim, H. Ryu, B. J. Moon, and J. Lee, "Clinical outcomes and prognostic factors in patients with myelopathy caused by thoracic ossification of the ligamentum flavum," Neurospine, vol. 15, no. 3, pp. 269-276, 2018.

[25] S. Yu, D. Wu, F. Li, and T. Hou, "Surgical results and prognostic factors for thoracic myelopathy caused by ossification of ligamentum flavum: posterior surgery by laminectomy," Acta Neurochirurgica, vol. 155, no. 7, pp. 1169-1177, 2013.

[26] T. Miyashita, H. Ataka, and T. Tanno, "Spontaneous reduction of a floated ossification of the ligamentum flavum after posterior thoracic decompression (floating method); report of a case (abridged translation of a primary publication)," The Spine Journal, vol. 13, no. 8, pp. e7-e9, 2013.

[27] N. S. Osman, Z. B. Cheung, A. K. Hussain et al., "Outcomes and complications following laminectomy alone for thoracic myelopathy due to ossified ligamentum flavum," Spine (Phila Pa 1976), vol. 43, no. 14, pp. E842-E848, 2018.

[28] R. Gao, W. Yuan, L. Yang, G. Shi, and L. Jia, "Clinical features and surgical outcomes of patients with thoracic myelopathy caused by multilevel ossification of the ligamentum flavum," The Spine Journal, vol. 13, no. 9, pp. 1032-1038, 2013.

[29] H. Hirabayashi, S. Ebara, J. Takahashi et al., "Surgery for thoracic myelopathy caused by ossification of the ligamentum flavum," Surgical Neurology, vol. 69, no. 2, pp. 114-116, 2008, discussion 116.

[30] S. He, N. Hussain, S. Li, and T. Hou, "Clinical and prognostic analysis of ossified ligamentum flavum in a Chinese population," Journal of Neurosurgery. Spine, vol. 3, no. 5, pp. 348354, 2005.

[31] T. Aizawa, T. Sato, H. Sasaki, T. Kusakabe, N. Morozumi, and S. Kokubun, "Thoracic myelopathy caused by ossification of the ligamentum flavum: clinical features and surgical results in the Japanese population," Journal of Neurosurgery. Spine, vol. 5, no. 6, pp. 514-519, 2006.

[32] N. Tsuzuki, S. Hirabayashi, R. Abe, and K. Saiki, "Staged spinal cord decompression through posterior approach for thoracic myelopathy caused by ossification of posterior longitudinal ligament," Spine (Phila Pa 1976), vol. 26, no. 14, pp. 1623-1630, 2001.

[33] B. Moal, V. Lafage, J. S. Smith et al., "Clinical improvement through surgery for adult spinal deformity: what can be expected and who is likely to benefit most?," Spine Deformity, vol. 3, no. 6, pp. 566-574, 2015. 